\title{
(S)- $\mathrm{N}$-Methyldihydroquinazolinones are the Active Enantiomers of Retro-2 Derived Compounds against Toxins
}

\author{
Neetu Gupta, ${ }^{\dagger, \|}$ Valérie Pons, ${ }^{\ddagger}, \|$ Romain Noël, ${ }^{\ddagger}$ David-Alexandre Buisson, ${ }^{\ddagger}$ Aurélien Michau, ${ }^{\dagger}$
} Ludger Johannes, ${ }^{\S, \perp}$ Daniel Gillet, ${ }^{\dagger} *$ Julien Barbier, ${ }^{\dagger}$ and Jean-Christophe Cintrat ${ }^{\ddagger} *$

${ }^{\dagger}$ CEA, iBiTec-S/SIMOPRO, CEA-Saclay, LabEx LERMIT, F-91191 Gif-sur-Yvette, France

${ }^{\ddagger}$ CEA, iBiTec-S/SCBM, CEA-Saclay, LabEx LERMIT, F-91191 Gif-sur-Yvette, France

${ }^{\S}$ U1143 INSERM, 75005 Paris, France

${ }^{\perp}$ Institut Curie, Centre de Recherche, Chemical Biology of Membranes and Therapeutic Delivery, 26 Rue d'Ulm, 75248 Paris Cedex 05, France

\section{Supporting Information}

ABSTRACT: This study reports the synthesis, chromatographic separation, and pharmacological evaluation of the two enantiomers of a new compound, named Retro2.1 , active against toxins by inhibiting intracellular trafficking via the retrograde route. The absolute configuration of the bioactive enantiomer has been assigned from X-ray diffraction to the $(S)$-enantiomer. To date, $(S)$-Retro- 2.1 is the most potent molecule to counteract the cytotoxic potential of ricin and Shiga toxin, with EC50 values of 23 and $54 \mathrm{nM}$, respectively.

KEYWORDS: Retro-2.1, Shiga toxin, ricin, dihydroquinazolinones

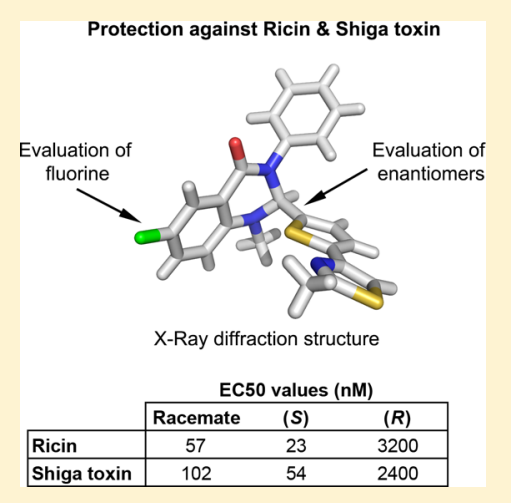

$\mathrm{R}$ icin is a highly toxic protein abundant in castor beans and classified as a potential bioweapon. After ingestion or inhalation, ricin causes major organ damages that can ultimately lead to the death of the exposed organism. To date no approved treatments or prophylaxis for human use is available. The Shiga toxin (Stx) family consists of related protein toxins that are produced by the bacteria Shigella dysenteriae and certain pathogenic strains of Escherichia coli. ${ }^{1,2}$ Bacteria producing these toxins are responsible for a number of diseases such as the life-threatening complication hemolytic uremic syndrome (HUS). ${ }^{3}$ In 2011, a major outbreak caused by E. coli O104:H4 infected about 4000 people in Europe, causing more than 900 cases of HUS resulting in 54 deaths. ${ }^{4}$ So far, no effective therapy exists for Stx intoxication. ${ }^{5,6}$ Ricin and Stx share several characteristics. They have one moiety (B-subunit) that binds to their respective cellular receptors (glycoproteins and glycolipids for ricin; the glycosphingolipid Gb3 for Stx), while another moiety (A-subunit) enters the cytosol and inactivates protein biosynthesis. Both toxins are transported in a retrograde manner from the plasma membrane via endosomes and the TGN to the endoplasmic reticulum (ER), ${ }^{7}$ before translocation to the cytosol, where they enzymatically inactivate the 28S RNA of the 60S ribosomal subunit (reviewed in refs $7-10)$. Inhibitors acting on the intracellular routing of these toxins likely offer novel options for the development of therapeutic strategies. ${ }^{11}$
We have recently identified a compound named Retro- 2 that protects human cells against ricin and Stx by blocking retrograde toxin transport at the early endosomes-TGN interface. $^{12}$ Retro-2 also protects mice against lethal ricin challenge. ${ }^{12}$ A structure-activity relationship (SAR) study of Retro-2 led to an improved activity against Stx up to 100-fold for compounds sharing a dihydroquinazolinone scaffold (cyclized Retro-2). ${ }^{13,14}$ We also demonstrated that only one enantiomer (compound $62,{ }^{13}$ Scheme 1 ) of dihydroquinazolinones was bioactive, but its absolute configuration was unsolved.

Here, we report the synthesis and evaluation of a new enantiopure dihydroquinazolinone compound, named Retro2.1, acting against Stx cytotoxicity with improved potency ( 500 -fold compared to Retro- 2$)$ and demonstrate that Retro2.1 is also effective against ricin in vitro $(\sim 1000$-fold increased activity compared to Retro-2). We establish from crystal $X$-ray diffraction data that the antitoxin activity resides mainly in the $S$-enantiomer.

During the course of our previous SAR study starting from Retro-2, we obtained $\mathrm{N}$-methylated cyclic analogues leading to

Received: November 12, 2013

Accepted: December 4, 2013

Published: December 4, 2013 
Scheme 1. Previous Studies on Retro-2 Optimization and Objectives of This Work (with EC 50 against Shiga Toxin)

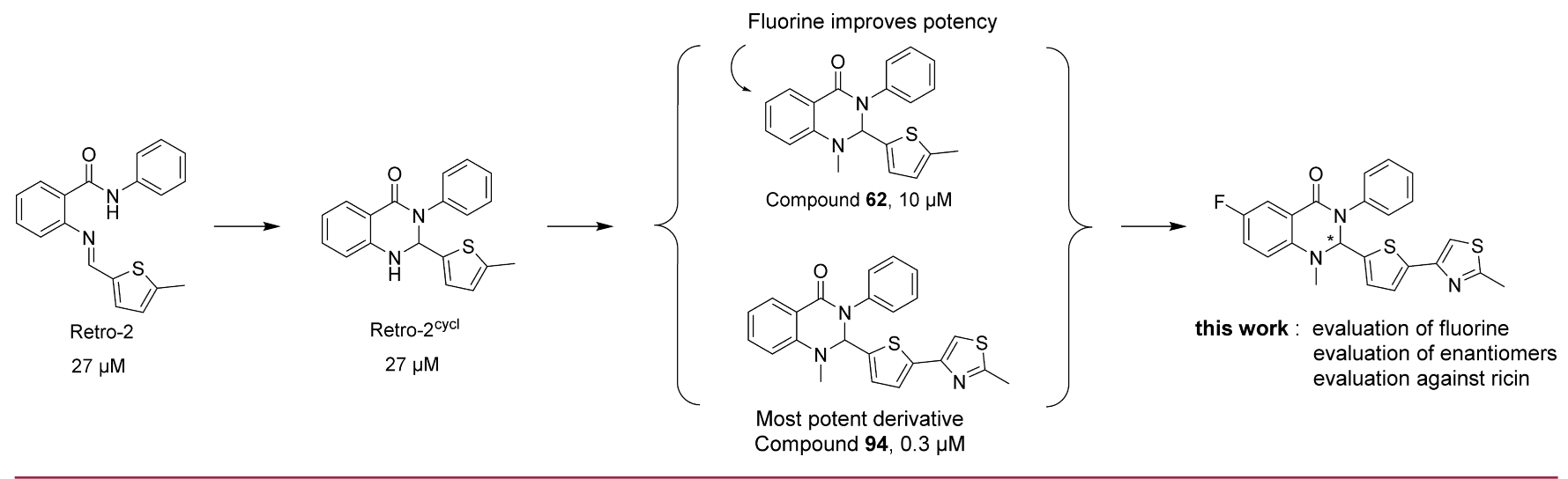

Scheme 2. Synthesis of 6-Fluoro-1-methyl-2-(5-(2-methylthiazol-4-yl)thiophen-2-yl)-3-phenyl-2,3-dihydroquinazolin-4(1H)one, Retro-2.1

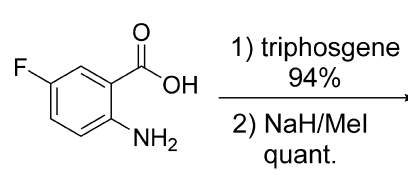

1

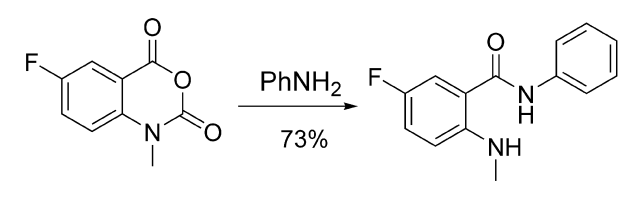

3

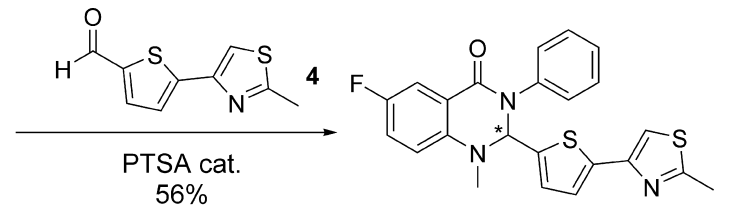

Retro-2.1, 5 a potent inhibitor of Stx action in cells (compound 94, $\mathrm{EC}_{50}=$ $0.3 \mu \mathrm{M},{ }^{13}$ Scheme 1). In parallel, substitution of the phenyl ring of the dihydroquinazolinone scaffold of 62 was evaluated. Among all the substituents, only the 6-fluoro derivative showed a better activity compared to the parent Retro- $2^{\text {cycl }}\left(\mathrm{EC}_{50}=11\right.$ $\mu \mathrm{M}$ vs $27 \mu \mathrm{M}$, respectively) ${ }^{13}$ (Scheme 1 ). We describe here the synthesis and evaluation of a compound combining the best pharmacophores.

The synthesis of compound $\mathbf{5}$ was achieved according to our previously published procedure starting from the commercially available 4-fluoro anthranilic acid 1 (Scheme 2) in 39\% yield over 4 steps.

In addition, since only one enantiomer was active on previous dihydroquinazolinones synthesized as racemates, ${ }^{13}$ a chiral phase HPLC separation of Retro-2.1 was performed on a ChiralPak IB column (see Experimental Section and Figure 1). No contamination of Retro-2.1a by Retro- $2.1 \mathrm{~b}$ or vice versa could be identified on the chromatograms (Figure 1).

To unequivocally assign the absolute configuration of each enantiomer, crystal structure determinations were obtained on separate enantiomers. Among the different crystallization

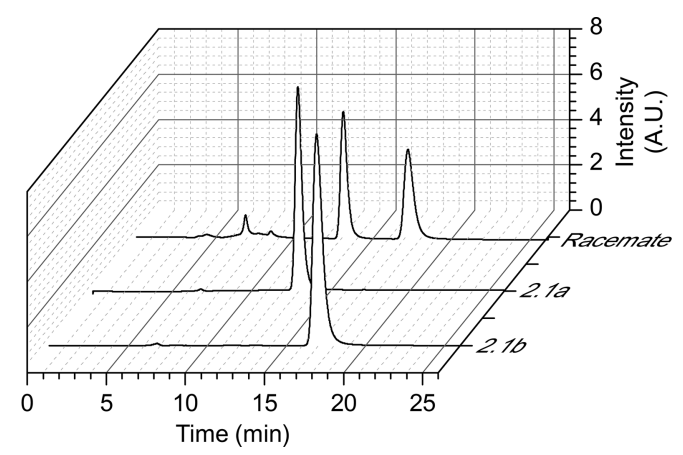

Figure 1. Chiral HPLC chromatograms of rac-Retro-2.1 and purified enantiomers, Retro-2.1a and Retro-2.1b. conditions that we screened, a mixture of $\mathrm{CH}_{2} \mathrm{Cl}_{2}$ and methanol (1:1) proved efficient to obtain monocrystals suitable for $X$-ray diffraction (slow evaporation method). These studies revealed that Retro-2.1a had a $S$-stereochemistry at the C-2 position of the dihydroquinazolinone core (Figure 2 ), while Retro- 2.1 b was the $R$-isomer.
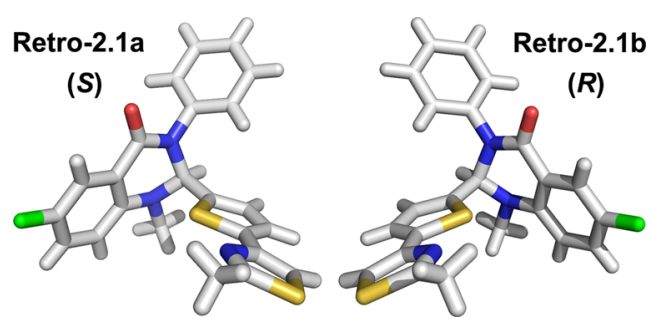

Figure 2. Structure determination of the Retro-2.1 enantiomers by Xray crystallography.

The biological activity of Retro-2.1 and each enantiomer was then evaluated against Stx and ricin (Figure 3). Retro-2.1 racemate inhibited Stx action on HeLa cells with an $\mathrm{EC}_{50}$ of $102 \pm 15.0 \mathrm{nM}$ (Figure 3, upper left panel; $n=9$ ) more potently than the previously described best compound (compound 94, ${ }^{13} \mathrm{EC}_{50}=0.3 \mu \mathrm{M}$ ). It also inhibited ricin induced-toxicity on human pulmonary A549 cells with an $\mathrm{EC}_{50}$ of $57 \pm 2.4 \mathrm{nM}$ (Figure 3, upper right panel; $n=3$ ).

When tested separately, Retro-2.1a $((S)$-Retro-2.1) potently inhibited toxin cytotoxicity (eutomer), while the second eluted compound, Retro-2.1b ( $(R)$-Retro-2.1), only showed moderate activity at micromolar concentrations (distomer). Indeed, (S)-Retro-2.1 inhibited both Stx cellular action $\left(\mathrm{EC}_{50}\right.$ of $54 \pm$ $16 \mathrm{nM}$; Figure 3, middle left panel; $n=7)$ and ricin $\left(\mathrm{EC}_{50}\right.$ of 23 $\pm 4 \mathrm{nM}$; Figure 3, middle right panel; $n=4$ ), whereas $(R)$-Retro-2.1 exhibited only an $\mathrm{EC}_{50}$ of $2.4 \pm 1 \mu \mathrm{M}$ against Stx (Figure 3, lower left panel; $n=4$ ) and an $\mathrm{EC}_{50}$ of $3.2 \pm 0.1 \mu \mathrm{M}$ against ricin (Figure 3, lower right panel; $n=3$ ). These results 
Shiga toxin

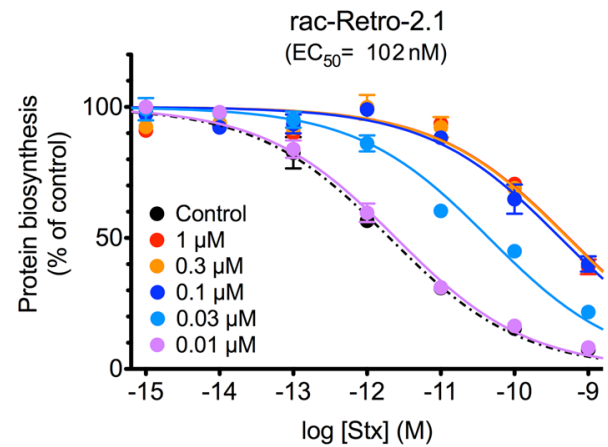

(S)-Retro-2.1
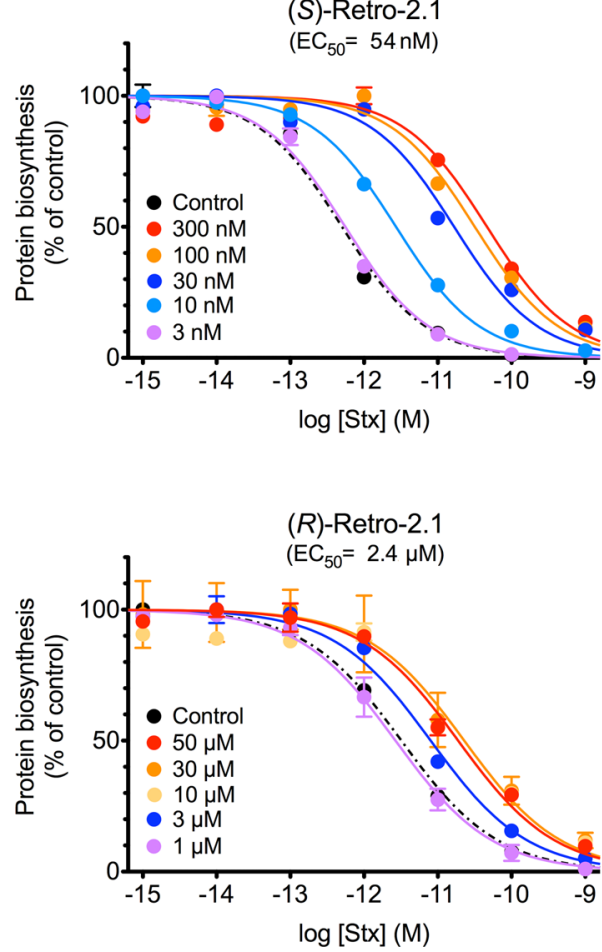

Ricin
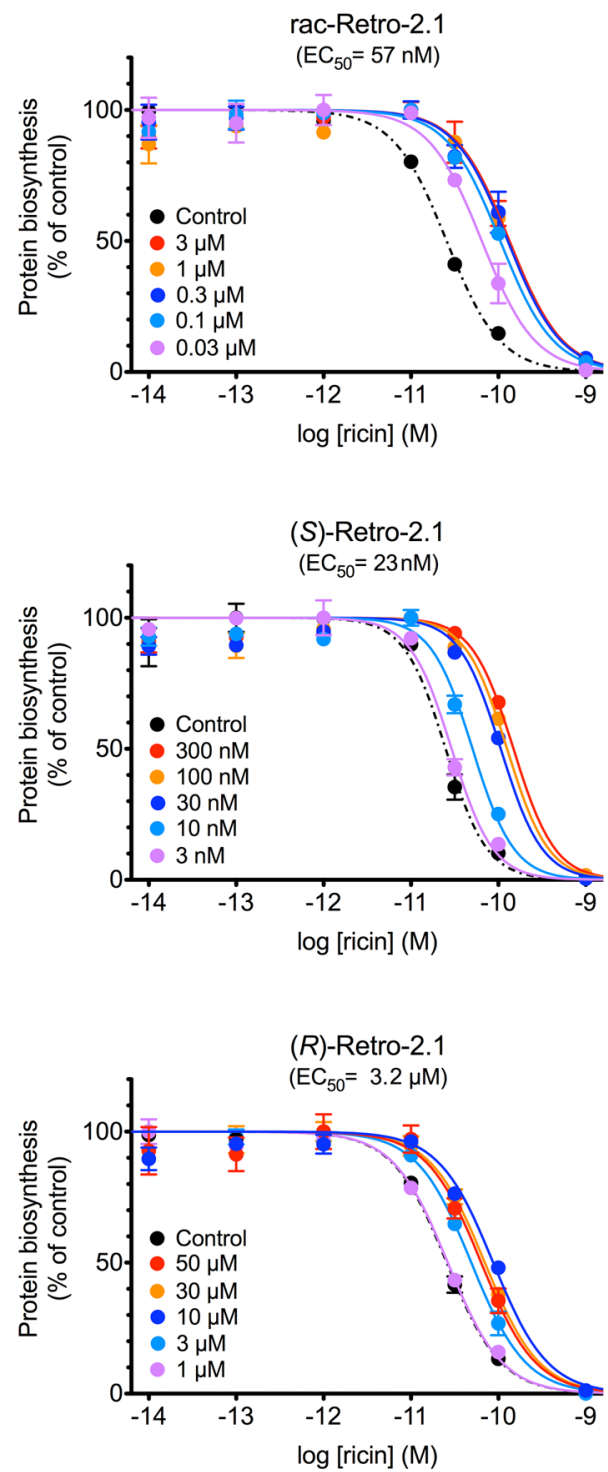

Figure 3. Retro-2.1 protects cells against Stx and ricin. Intoxication of HeLa cells by Stx (left) and intoxication of A549 cells by ricin (right) in the presence of inhibitors. Cells were incubated $1 \mathrm{~h}$ at $37{ }^{\circ} \mathrm{C}$ with DMSO $(0.04 \%$, black data points in all parts of the figure), rac-Retro-2.1 (various concentrations; upper panel), ( $S$ )-Retro-2.1 enantiomer (various concentrations; middle panel), or $(R$ )-Retro-2.1 enantiomer (various concentrations; lower panel) before addition of toxins at the indicated concentrations. Each data point represents the mean of duplicate \pm SEM of a representative experiment. $\mathrm{EC}_{50}$ values are the mean of $n$ independent experiments (see text for more details). Note that the concentrations used for racemate and enantiomers are different.

are in accordance with the $\mathrm{EC}_{50}$ values obtained from the racemate form with a 2 -fold increase of potency of the eutomer. Eudismic ratios of 45 and 135, respectively for Stx and ricin, were calculated from the $\mathrm{EC}_{50}$ values of cytotoxicity assays reported in this paper, reflecting a high enantioselectivity. This eudismic ratio may also explain the absence of activity of the distomer of compound 62 reported previously ${ }^{13}$ on Stx, since the highest concentration tested for the distomer was $30 \mu \mathrm{M}$ (due to low solubility) while the $\mathrm{EC}_{50}$ of the racemate was 10 $\mu \mathrm{M}$.

It is worth noting that besides a lower potency, determined by a higher $\mathrm{EC}_{50},(R)$-Retro-2.1 also displayed a reduced efficacy (lower maximal $R$-value) in comparison to ( $S$ )-Retro2.1 against both Stx and ricin (for Stx, $R_{(S)}=357$ vs $R_{(R)}=68, n$ $=6$; for ricin, $R_{(S)}=5$ vs $R_{(R)}=2.9, n=3 ; R$ values are from experiments performed in parallel). These differential pharmacological activities proved that only the $(S)$-enantiomer fully retained the desired antitoxin activity.

Retro-2.1 has a stronger efficacy ( $R$-values) against Stx than against ricin, as observed for Retro- $2 .^{12}$ Most likely, ricin can use several mechanisms to traffic into cells by binding to a variety of glycoreceptors. In contrast, Stx only binds to Gb3 and may be facing limited molecular redundancy for reaching TGN membranes. Ricin has also been proposed to cross endosomal membranes to reach the cytosol, as an alternative mechanism to retrotranslocation from the endoplasmic reticulum. ${ }^{15}$ This type of endosomal escape would allow bypassing a Retro-2.1induced retrograde transport block.

We describe herein the synthesis and chromatographic separation of Retro-2.1 enantiomers. In vitro experiments 
proved the configuration of the stereocenter in position 2 is crucial for activity, since one enantiomer is active to protect cells against Stx and ricin. The absolute stereochemistry of the eutomer has been assigned by X-ray diffraction. The $(S)$-isomer proved to be the bioactive enantiomer, and $(S)$-Retro-2.1 is the most active compound against these toxins reported so far.

Since $N$-methyldihydroquinazolinones derived from Retro- 2 block the retrograde pathway within cells, optimized $(S)$-Retro2.1 has the potential to be developed as a broad-spectrum antidote to a wide array of pathogens, including toxins and parasites, as demonstrated previously in vitro and in vivo for Retro-2. ${ }^{12,16}$

\section{ASSOCIATED CONTENT}

\section{S Supporting Information}

General procedures, characterization of new compounds, crystal structure determination of compound 5, chemicals for in vitro experiments, intoxication assays, and determination of $\mathrm{EC}_{50}$ values. This material is available free of charge via the Internet at http://pubs.acs.org.

\section{AUTHOR INFORMATION}

\section{Corresponding Authors}

*For D.G.: phone, + 331690876 46; fax, + 331690890 71; e-mail, daniel.gillet@cea.fr.

*For J.-C.C.: phone, + 331690821 07; fax, + 331690879 91; e-mail, jean-christophe.cintrat@cea.fr.

\section{Author Contributions}

${ }$ N.G. and V.P. contributed equally to this work.

\section{Funding}

This work (Project RetroScreen) has been funded by the French National Agency for Research (ANR) under Contract ANR-11-BSV2-0018, the Joint ministerial program of R\&D against CBRNe risks and CEA. N.G. was funded by the CEA international $\mathrm{PhD}$ program.

\section{Notes}

The authors declare no competing financial interest.

\section{ABBREVIATIONS}

Stx, Shiga toxin; E. coli, Escherichia coli; HUS, hemolytic uremic syndrome; Gb3, globotriaosylceramide; ER, endoplasmic reticulum; RNA, ribonucleic acid; SAR, structure-activity relationship; $\mathrm{EC}_{50}, 50 \%$ effective concentration; HPLC, high performance liquid chromatography; TGN, trans-Golgi network; DMSO, dimethyl sulfoxide; SEM, standard error of the mean; RP-HPLC, reversed phase-high performance liquid chromatography; THF, tetrahydrofuran; DMF, dimethyl formamide; PTSA, para-toluene sulfonic acid; NMR, nuclear magnetic resonance; MS, mass spectrometry; HRMS, high resolution mass spectrometry; LC/MS, liquid chromatography coupled to mass spectrometry; IR, infrared; UV, ultraviolet; DAD, diode array detector; DMEM, Dulbecco's modified Eagle's medium; $\mathrm{IC}_{50}, 50 \%$ inhibition concentration; TLC, thin-layer chromatography

\section{REFERENCES}

(1) Acheson, D. W. K.; Keusch, G. T. The family of Shiga toxins. In The comprehensive sourcebook of bacterial protein toxins, 2nd ed.; Alouf, J. E.; Freer, J. H., Eds.; Elsevier: Paris, 1999; pp 229-242.

(2) O'Brien, A. D.; Holmes, R. K. Shiga and Shiga-like toxins. Microbiol. Rev. 1987, 51, 206-220.
(3) Tarr, P. I.; Gordon, C. A.; Chandler, W. L. Shiga-toxin-producing Escherichia coli and haemolytic uraemic syndrome. Lancet 2005, 365, 1073-1086.

(4) Karch, H.; Denamur, E.; Dobrindt, U.; Finlay, B. B.; Hengge, R.; Johannes, L.; Ron, E. Z.; Tønjum, T.; Sansonetti, P. J.; Vicente, M. The enemy within us: lessons from the 2011 European Escherichia coli O104:H4 outbreak. EMBO Mol. Med. 2012, 4, 841-848.

(5) Tarr, P. I.; Sadler, J. E.; Chandler, W. L.; George, J. N.; Tsai, H. M. Should all adult patients with diarrhoea-associated HUS receive plasma exchange? Lancet 2012, 379, 516 (author reply pp 516-517).

(6) Menne, J.; Nitschke, M.; Stingele, R.; Abu-Tair, M.; Beneke, J.; Bramstedt, J.; Bremer, J. P.; Brunkhorst, R.; Busch, V.; Dengler, R.; Deuschl, G.; Fellermann, K.; Fickenscher, H.; Gerigk, C.; Goettsche, A.; Greeve, J.; Hafer, C.; Hagenmuller, F.; Haller, H.; HergetRosenthal, S.; Hertenstein, B.; Hofmann, C.; Lang, M.; Kielstein, J. T.; Klostermeier, U. C.; Knobloch, J.; Kuehbacher, M.; Kunzendorf, U.; Lehnert, H.; Manns, M. P.; Menne, T. F.; Meyer, T. N.; Michael, C.; Munte, T.; Neumann-Grutzeck, C.; Nuernberger, J.; Pavenstaedt, H.; Ramazan, L.; Renders, L.; Repenthin, J.; Ries, W.; Rohr, A.; Rump, L. C.; Samuelsson, O.; Sayk, F.; Schmidt, B. M.; Schnatter, S.; Schocklmann, H.; Schreiber, S.; von Seydewitz, C. U.; Steinhoff, J.; Stracke, S.; Suerbaum, S.; van de Loo, A.; Vischedyk, M.; Weissenborn, K.; Wellhoner, P.; Wiesner, M.; Zeissig, S.; Buning, J.; Schiffer, M.; Kuehbacher, T. Validation of treatment strategies for enterohaemorrhagic Escherichia coli O104:H4 induced haemolytic uraemic syndrome: case-control study. BMJ [Br. Med. J.] 2012, 345, e4565.

(7) Johannes, L.; Popoff, V. Tracing the retrograde route in protein trafficking. Cell 2008, 135, 1175-1187.

(8) Watson, P.; Spooner, R. A. Toxin entry and trafficking in mammalian cells. Adv. Drug. Delivery Rev. 2006, 58, 1581-1596.

(9) Sandvig, K.; Spilsberg, B.; Lauvrak, S. U.; Torgersen, M. L.; Iversen, T. G.; van Deurs, B. Pathways followed by protein toxins into cells. Int. J. Med. Microbiol. 2004, 293, 483-490.

(10) Lord, J. M.; Roberts, L. M.; Lencer, W. I. Entry of protein toxins into mammalian cells by crossing the endoplasmic reticulum membrane: co-opting basic mechanisms of endoplasmic reticulumassociated degradation. Curr. Top. Microbiol. Immunol. 2005, 300, 149-168.

(11) Barbier, J.; Bouclier, C.; Johannes, L.; Gillet, D. Inhibitors of the cellular trafficking of ricin. Toxins (Basel) 2012, 4, 15-27.

(12) Stechmann, B.; Bai, S. K.; Gobbo, E.; Lopez, R.; Merer, G.; Pinchard, S.; Panigai, L.; Tenza, D.; Raposo, G.; Beaumelle, B.; Sauvaire, D.; Gillet, D.; Johannes, L.; Barbier, J. Inhibition of retrograde transport protects mice from lethal ricin challenge. Cell 2010, 141, 231-242.

(13) Noel, R.; Gupta, N.; Pons, V.; Goudet, A.; Garcia-Castillo, M. D.; Michau, A.; Martinez, J.; Buisson, D. A.; Johannes, L.; Gillet, D.; Barbier, J.; Cintrat, J. C. N-Methyldihydroquinazolinone derivatives of Retro-2 with enhanced efficacy against Shiga Toxin. J. Med. Chem. 2013, 56, 3404-3413.

(14) Park, J. G.; Kahn, J. N.; Tumer, N. E.; Pang, Y. P. Chemical structure of Retro-2, a compound that protects cells against ribosomeinactivating proteins. Sci. Rep. 2012, 2, 631.

(15) Beaumelle, B.; Alami, M.; Hopkins, C. R. ATP-dependent translocation of ricin across the membrane of purified endosomes. J. Biol. Chem. 1993, 268, 23661-23669.

(16) Canton, J.; Kima, P. E. Targeting host syntaxin-5 preferentially blocks Leishmania parasitophorous vacuole development in infected cells and limits experimental Leishmania infections. Am. J. Pathol. 2012, 181, 1348-1355. 